\title{
Visualizing Participatory Inequities in Classroom Data
}

\author{
Poorna Talkad Sukumar* \\ University of Notre Dame
}

\author{
Daniel Reinholz \\ San Diego State University
}

\author{
Niral Shah \\ University of Washington
}

\author{
Aaron Striegel ${ }^{\S}$ \\ University of Notre Dame
}

\begin{abstract}
Classroom participation data, disaggregated by race and gender, can enable teachers to reflect on their implicit biases and make informed changes to their practices. In this design study, we collaborated with researchers in education to explore a design space for representing participatory inequities in classroom data. This paper contributes to the relatively new area of addressing implicit biases using visualizations and more broadly, aims at improving equity in classrooms.
\end{abstract}

\section{INTRODUCTION}

Student participation in classrooms is strongly linked to their learning and identity development [3]. However, research shows that marginalized students, for example, women in mathematics discipline, typically get fewer opportunities to participate in classrooms and hence, fewer learning opportunities [3]. Therefore, it is important for teachers to consciously enforce participatory equity in their classrooms to ensure that students, especially those belonging to marginalized student groups, get a fair share of participation opportunities.

Talk-based participation in classrooms is quantifiable and can provide teachers with actionable data [3]. This data, disaggregated by various social markers including race and gender, can be used to make teachers aware of their implicit biases and inform their practices. Implicit or social biases refer to unconscious, ingrained attitudes and stereotypes about groups of people [6].

Visualizing participatory inequities, that is, disparities in classroom participation, to make teachers aware of their implicit biases can be challenging. Visualization idioms, by definition, are each effective for specific tasks and can be ill-suited for other tasks [2](Ch. 1 ). To this end, a visual analytics tool, known as EQUIP ${ }^{1}$, encompassing a range of visualization idioms was developed to enable teachers to explore and reflect on different representations of their classroom data. Preliminary validation studies with EQUIP, however, produced mixed results and showed that there was room for improvement.

In this work, we present aspects of the core design phase [5] in our design study where we, in collaboration with domain experts, consider potential solutions for visualizing class participation data informed by visualization design principles and guidelines. This work contributes to the upcoming area of addressing social biases using visualizations and more broadly, can be useful for reducing inequities in teaching practices.

\section{Design: Data/Task abstraction and Visual En- CODING/INTERACTION}

We present below descriptions of the data and tasks and a subset of the visualization solutions we generated.

\footnotetext{
*e-mail: ptalkads@nd.edu

†e-mail: daniel.reinholz@sdsu.edu

Fe-mail: niral@uw.edu

$\S$ e-mail: striegel@nd.edu
}

\footnotetext{
${ }^{1}$ https:www. equip.ninja
}

\subsection{Data Description}

Classroom data corresponding to a teacher is generated by videotaping the teacher's classrooms and then manually coding the videos [3]. Each instance of student participation in the classroom, called a "contribution", is described using various qualitative dimensions pertaining both to the quality of student participation and the quality of teacher interaction. The coding is performed by multiple coders ensuring high interrater reliability. These codes are then combined with student demographics, such as race and gender, to generate the visualizations. The codes have also been applied across disciplines and also across different education levels, ranging from elementary school to the university-level [4].

The dataset can be viewed as a static table where the rows represent individual student contributions and columns include the qualitative dimensions and demographic attributes. All of the qualitative dimensions and demographic attributes are categorical variables.

\subsection{Task: Compare Contributions}

The domain experts outlined specific tasks that they expect teachers to perform with the visualizations in order to understand the data and reflect on their biases. These tasks include:

- Comparing contributions across the different student groups; e.g., do Black students participate as much as White students?

- Comparing contributions among individual students; e.g., are there students who didn't participate at all? and are there some individual students who dominated the class participation?

In addition to looking at the quantity of contributions, these tasks are then applied to also look at the quality of contributions for the different groups and students. These tasks are designed to help the teachers identify when marginalized students and student groups get less than a fair share of participation opportunities.

We referred to the visualization design book by Munzner [2] as well as various chart choosers and reference guides to select suitable visual encodings. We considered "magnitude" and "part-to-whole" charts including bar charts, stacked bars, dot arrays, isotypes, and grid plots to represent the data (see Figure 1(a)-(d)). Isotypes and grid plots can especially be good solutions when representing whole numbers.

Various interaction strategies can be used to link, coordinate, and arrange these multiple views on the interface to facilitate exploration of the multivariate data [2](Ch. 12). For example, Figure 1(b) shows the use of small multiples to present details of the contribution types within a demographic. In isotype and dot array representations, each icon/dot can be used to represent a student whose name is displayed on mouse-hover (see Figure 1(d)). We also considered including options to filter and aggregate attributes within a view [2](Ch. 13).

\subsection{Derived data: Equity Ratio}

The domain experts also use a derived metric called Equity Ratio which is intended to communicate participatory equity in a simplified manner. For each demographic group, the equity ratio is calculated as the ratio between the percentage of contributions of that group and the percentage of the demographic within the classroom. For example, if $50 \%$ of the class is comprised of male students and their contributions make up $75 \%$ of the total class participation, then 

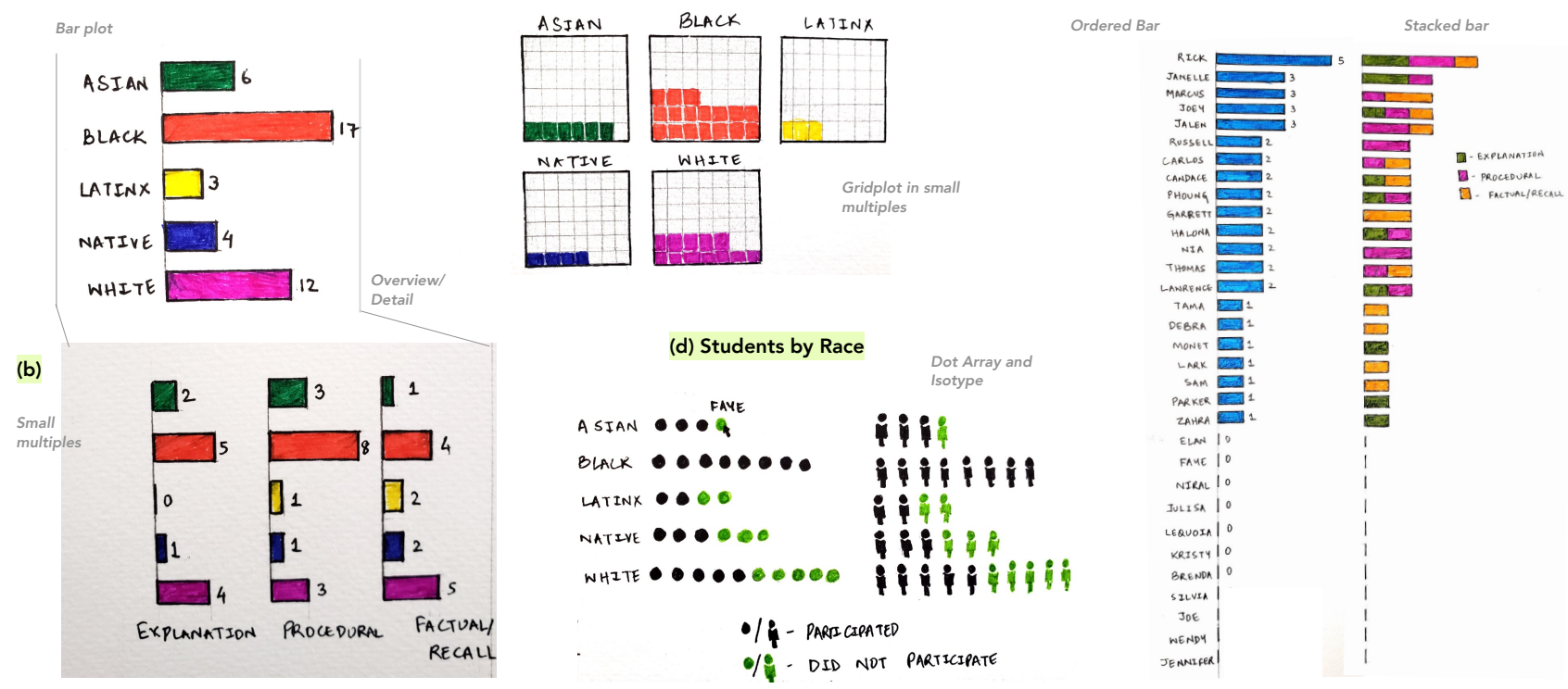

(e) Representations for Equity Ratio
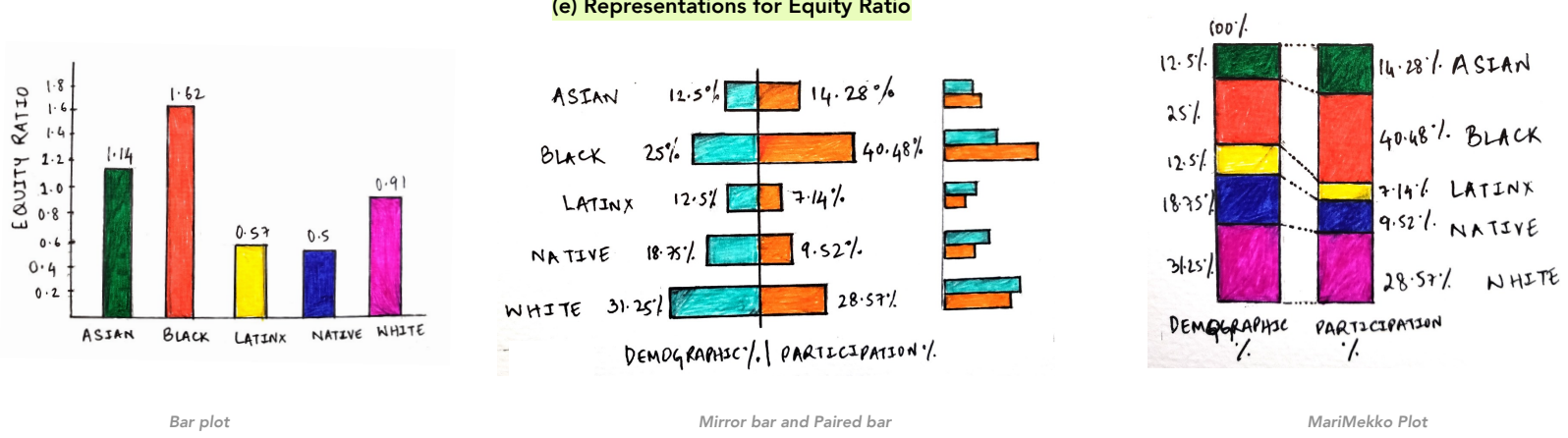

Figure 1: Sample design sketches for visualizing classroom participation data. A synthetic dataset [1] was used to create the visualizations.

the equity ratio for male students would be $1.5(75 / 50)$ which is a relatively high rate of participation.

The interpretation of equity ratio can be non-trivial. While the equity ratio uses a demographic's percentage in the classroom as a baseline to compare the demographic's participation, it does not suggest that the two percentages should be proportional. The equity ratio is instead intended to highlight when certain marginalized student groups have a disproportionately low level of participation. We considered the following representations for equity ratio (see Figure 1(e)):

(1) representing the equity ratio directly, e.g, by using bar plot;

(2) representing the individual percentages in the equity ratio instead of the equity ratio to prevent potential emphasis on the "equity ratio $=1.0$ " metric in (1), e.g., by using mirror bar and paired bar; and (3) representing the zero-sum nature of participation to illustrate that an increase in one group's participation implies a decrease in participation in other group(s), e.g., by using a MariMekko plot.

\section{Conclusion}

Designing visualizations for addressing implicit biases can be nontrivial and entails going beyond selecting suitable encodings because of the inherent subjective nature of the biases. Certain visualizations, despite being theoretically good solutions, can potentially cause some teachers to make less equitable decisions. Hence the proposed design solutions have to be rigorously validated before they are used in practice.

\section{ACKNOWLEDGMENTS}

This material is based upon work supported by the National Science Foundation under Grant No. 1943146

\section{REFERENCES}

[1] B. Herbel-Eisenmann and N. Shah. Detecting and reducing bias in questioning patterns. Mathematics Teaching in the Middle School, 24(5):282289, 2019.

[2] T. Munzner. Visualization analysis and design. CRC press, 2014.

[3] D. L. Reinholz and N. Shah. Equity analytics: A methodological approach for quantifying participation patterns in mathematics classroom discourse. Journal for Research in Mathematics Education, 49(2):140177, 2018.

[4] D. L. Reinholz, A. Stone-Johnstone, and N. Shah. Walking the walk: using classroom analytics to support instructors to address implicit bias in teaching. International Journal for Academic Development, pp. 1-14, 2019.

[5] M. Sedlmair, M. Meyer, and T. Munzner. Design study methodology: Reflections from the trenches and the stacks. IEEE transactions on visualization and computer graphics, 18(12):2431-2440, 2012.

[6] K. Yogeeswaran, T. Devos, and K. Nash. Understanding the nature, measurement, and utility of implicit intergroup biases. 2017. 